\title{
Versatile Fluidic Cell for In-situ Electrochemical Measurements in SEM
}

J. Velmurugan ${ }^{1,2}$, A. Stevanovic ${ }^{1,2}$, F. Yi $^{3}$, D. LaVan ${ }^{3}$ and A. Kolmakov ${ }^{1}$

${ }^{1}$ Center for Nanoscale Science and Technology, NIST, Gaithersburg, MD, USA

2. Maryland Nanocenter, University of Maryland, College park, MD, USA

${ }^{3 .}$ Materials Measurement Laboratory, NIST, Gaithersburg, MD, USA

Electrochemical techniques such as electrodeposition are crucial for fabrication of nanostructured materials. The details of cluster nucleation and growth at early stages of electrodeposition are extremely important for morphology and properties of the deposits. The growth mechanism is usually determined by analyzing global electrochemical data in conjunction with ex-situ images. Though this approach gives us a clear picture as a whole, it fails to reveal the evolution of islands morphology at nanoscale and its effects on reaction rate. Lately, in-situ transmission electron microscopy (TEM) in liquids has been used successfully to study the surface and interface processes and also to interpret the electrochemical data [1-3]. Surprisingly, there has been less research performed using liquid scanning electron microscopy (SEM) [4]. In this report, we describe novel chip and cell designs enabling us to study electrochemical processes in real time and as a function of temperature using SEM in liquids.

The cell is shown in figure 1. The cell is based on a microfabricated Si chip equipped with $50 \mathrm{~nm}$ thick SiNx membrane, $150 \mathrm{~nm}$ thick Pt electrodes as working electrodes (WE) and heaters [5-6]. $30 \mathrm{~nm}$ layer of $\mathrm{Al} 2 \mathrm{O} 3$ covers the top of electrodes and heaters and serves as an electrochemical protection layer. In conventional electrochemical experiments, a three-electrode geometry is usually preferred. This is because even if the counter electrode (CE) is designed to be larger than the working electrode, the current passing the cell will polarize the counter electrode. If a third electrode is used as a pseudo reference electrode (RE), the current drawn from it should be extremely small (e.g., a few fA) to avoid similar polarization issues. In addition, monitoring the voltage of the counter electrode proves to be useful even in three-electrode experiments to avoid large voltage sweeps that could damage the counter electrode or cause bubble formation in the electrolyte. Both the counter electrode $(\mathrm{Pt})$ and the reference electrode (Ag) were wire bonded to the ceramic chip carrier. The ceramic microchip carrier pressed against a rubber gasket sits tightly on the top of a fluidic cell made of polyetheretherketone. For Ag electroplating, $100 \mathrm{~mol} / \mathrm{m} 3$ solution of $\mathrm{AgNO} 3$ in water was introduced through syringe and the cell was sealed.

Figure 2 shows the typical SEM images obtained during in-situ Ag electrodeposition on Pt electrode. The images detail the morphological evolution of silver dendrites that were prepared at $100 \mathrm{~mol} / \mathrm{m} 3$ silver nitrate aqueous solution. The growth of the dendritic nanostructure was carefully monitored in real time by a current measurement. As the applied deposition potential of $-0.5 \mathrm{~V}$, the initial silver nucleation experienced a morphological evolution and converted to some widespread dendrites. In the first few seconds, Ag start nucleating at several areas of Pt electrode. As the reaction proceeded individual dendrite length of about $5 \mu \mathrm{m}$ to $10 \mu \mathrm{m}$ start branching out from the electrode.

In conclusion, the proposed environmental cell is well suited for in-situ electrochemistry. The setup can be widely extended to investigate morphological transformations in biological systems and electrochemical phenomena like corrosion.

References:

[1] A. Radisic et al, Nano Letters, 6(2) (2006), p. 238. 
[2] N. de Jonge and F. M. Ross, Nature Nanotechnology, 6(11) (2011), p. 695.

[3] J. M. Grogan et al, Journal of the Indian Institute of Science, 92 (2)(2012), p. 295.

[4] E. Jensen et al, Ultramicroscopy, volume 129 (2013), p. 63-69

[5] F. Yi, W. Osborn, J. Betz, J and D.A. LaVan Microelectromechanical Systems, 24 (2015), p. 1185.

[6] M. D. Grapes, et al, Review of Scientific Instruments, 85 (2014), p. 084902.
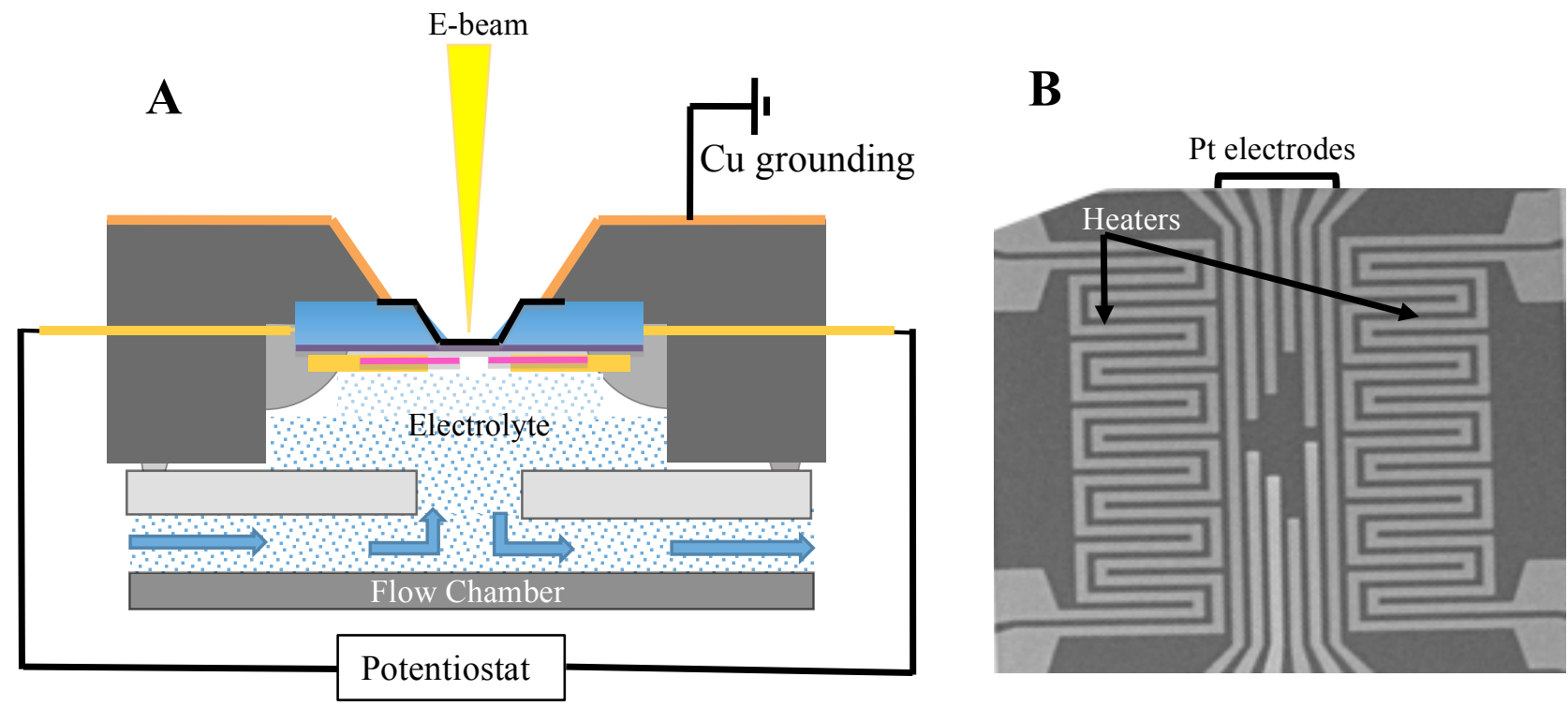

Figure 1. A. Microchip holder and liquid cell. The microchip carrier (dark grey) pressed against a rubber gasket sits tightly on the top of a liquid cell (gray). The chip carrier assembly consists of a Si-chip that hosts a metal oxide nanowire which can experience different gas environments (as shown in B in the circled area.) B. Design of a microchip. The microchip consists of Pt electrodes and Pt heaters. The Pt electrodes are the working electrodes in electrochemical process and the Pt heaters allow for uniform heating and cooling.
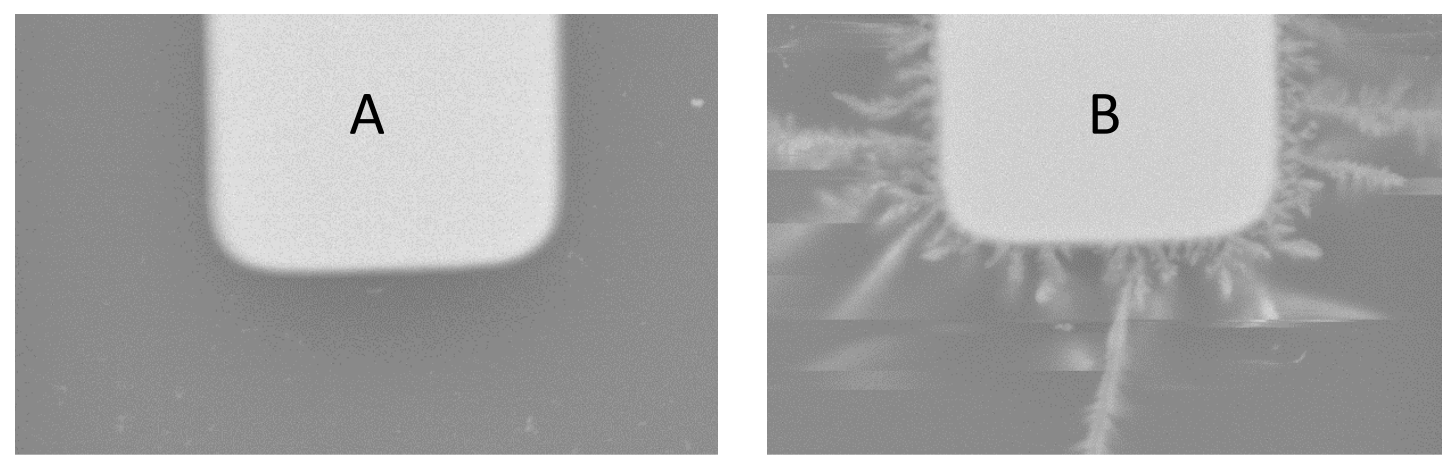

Figure 2. SEM images of the Ag dendrites electrochemically grown over at Pt electrode before (A) and after (B) potential hold at $-0.5 \mathrm{~V}$ vs $\mathrm{Ag} / \mathrm{AgCl}$ in $100 \mathrm{~mol} / \mathrm{m}^{3} \mathrm{AgNO}_{3}$ water solution through $50 \mathrm{~nm}$ SiN membrane 